\title{
SARS-CoV-2 (COVID-19) en gestación y placenta: una revisión narrativa sobre el estado del arte
}

\section{SARS-CoV-2 (COVID-19) in pregnancy and placenta: a narrative review on state of the art}

\section{Marcelo González1,2*, Felipe Troncoso ${ }^{3}$ y Carlos Escudero 2,3}

${ }^{1}$ Laboratorio de Investigación Materno-Fetal (LIMaF), Departamento de Obstetricia y Ginecología, Facultad de Medicina, Universidad de Concepción, Concepción; ${ }^{2}$ Group of Research and Innovation in Vascular Health (GRIVAS Health), Chillán; ${ }^{3}$ Laboratorio de Fisiología Vascular, Departamento de Ciencias Básicas, Universidad del Bío-Bío, Chillán. Chile

\section{Resumen}

La crisis sanitaria por la pandemia de COVID-19, enfermedad generada por la infección con el SARS-CoV-2, ha llevado a la pérdida de más de 25,000 vidas en Chile, con más de 370,000 mujeres entre 15 y 44 años con infección confirmada. Se ha reportado una mayor vulnerabilidad de las mujeres gestantes en cuanto a desarrollar cuadros de COVID-19 graves o críticos, con un aumento de la incidencia de resultados obstétricos y perinatales adversos. Es relevante considerar que un alto porcentaje de las gestantes infectadas con SARS-CoV-2 son asintomáticas, lo cual nos pone en alerta en cuanto a que ciertos efectos del virus durante la gestación podrían no ser evidentes para la observación clínica. Se ha demostrado la presencia de SARSCoV-2 en la placenta, asociándose la infección placentaria con alteraciones vasculares que podrían afectar el flujo útero-placentario. Por otro lado, la transmisión vertical al feto parece que es poco frecuente, pero factible. Se resumen las evidencias disponibles hasta el momento sobre los principales efectos de la COVID-19 en la gestación, con énfasis en los estudios sobre los efectos de la infección por SARS-CoV-2 en la placenta. El objetivo es relevar el tema, destacando que son diversas las preguntas que necesitan ser abordadas, considerando el impacto que esta pandemia podría tener sobre la salud gestacional.

Palabras clave: COVID-19. SARS-CoV-2. Gestación. Placenta.

\section{Abstract}

In Chile, the COVID-19 pandemic, a disease induced by infection with SARS-CoV-2, has caused more than 25,000 deaths. More than 370,000 women between 15 and 44 years have been detected with the infection. The greater vulnerability of pregnant women has been reported, mainly related to a higher risk for severe or critical COVID-19, with an increased incidence of adverse obstetrics and perinatal outcomes. It is relevant to consider that a high percentage of pregnant women infected with SARS-CoV-2 are asymptomatic for COVID-19, which indicates that specific effects of the virus during pregnancy may not be evident from clinical observation. The presence of SARS-CoV-2 in the placenta has been demonstrated, associating placental infection with vascular alterations that could affect utero-placental flow. On the other hand, vertical transmission to the fetus is rare but feasible. This manuscript summarizes the evidence available to date on the main effects of COVID-19 in pregnancy, emphasizing studies about the impact of SARS-CoV-2 in the placenta. This review aims to promote this issue, highlighting that several questions need to be addressed, considering the effect this pandemic could have on gestational health.

Key words: COVID-19. SARS-CoV-2. Pregnancy. Placenta.

Correspondencia:

*Marcelo González

E-mail: mgonzalezo@udec.cl
Disponible en internet: 27-10-2021

\section{Rev Chil Obstet Ginecol. 2021;86(4):425-432}

www. rechog.com 


\section{Introducción}

Los primeros casos de COVID-19 se registraron en la ciudad de Wuhan, China, en el mes de diciembre de 2019. En el fluido broncoalveolar de los pacientes fue posible aislar e identificar el virus que genera esta enfermedad, que fue llamado SARS-CoV-2, perteneciente al género betacoronavirus, del cual forman parte los virus de origen zoonótico SARS-CoV (coronavirus causante de síndrome agudo respiratorio) y MERSCoV (coronavirus relacionado con el síndrome respiratorio del Medio Oriente) ${ }^{1}$. El virus se propagó rápidamente, recibiendo la categoría de pandemia por parte de la Organización Mundial de la Salud ${ }^{2}$ en el mes de marzo de $2020^{3}$. En Chile se han registrado más de 1 millón de contagios (de acuerdo con el reporte diario MINSAL del 25/04/20214) y más de 25,000 muertes por COVID-19 confirmada y 7096 muertes sospechosas de COVID-19 (con sintomatología relacionada), según los registros del Departamento de Estadística e Información en Salud (DEIS) del Ministerio de Salud (MINSAL) 5 . Las mujeres gestantes han mostrado ser vulnerables a la enfermedad, lo cual queda demostrado por una encuesta realizada por la Sociedad Chilena de Obstetricia y Ginecología (SOCHOG), en la que se recolectó información de 7638 partos atendidos entre el 1 de marzo y el 15 de abril del 2021. En este grupo, un 30\% (115) de las pacientes requirieron hospitalización en la unidad de cuidados intensivos (UCI), y de estas, un 58\% (66) requirieron ventilación asistida ${ }^{6}$.

Durante la gestación humana se producen adaptaciones fisiológicas maternas que involucran a los sistemas respiratorio, cardiovascular, endocrino e inmunitario ${ }^{7}$, entre otros. Para sostener el desarrollo fetal se requieren aumentos significativos de la frecuencia respiratoria, el gasto cardiaco y la volemia, y una disminución también significativa de la resistencia vascular sistémica ${ }^{8}$. Estas complejas adaptaciones fisiológicas hacen suponer que la gestación podría aumentar la susceptibilidad a contraer la infección por SARS-CoV-2 o desarrollar cuadros graves de COVID$19^{9}$, especialmente según los reportes de muertes en mujeres gestantes asociadas a COVID-19 en Brasil $^{10} y$ México ${ }^{11}$. El primer reporte sobre COVID-19 en el embarazo de los Centers for Disease Control and Prevention de los Estados Unidos de América mostró que las mujeres de origen hispano y afroamericano presentaban mayor riesgo de infección por SARSCoV-2, y que en general las mujeres gestantes tenían mayor riesgo de desarrollar un cuadro grave o crítico de COVID-19 ${ }^{12}$. Información reciente confirma que el desarrollo de cuadros sintomáticos de COVID-19 aumenta la tasa de mortalidad en mujeres gestantes en comparación con no gestantes, además de inducir resultados obstétricos y perinatales adversos ${ }^{13,14}$. Por otro lado, aparte de los efectos que tiene la COVID-19 sobre la salud materna, se ha puesto atención en la posibilidad de transmisión del virus de la madre al feto in utero (transmisión vertical) ${ }^{15}$.

Todos estos temas son tratados en la presente revisión, con un enfoque centrado en los trabajos publicados que han estudiado específicamente la presencia de SARS-CoV-2 en la placenta y sus potenciales efectos sobre la función placentaria.

\section{Impacto de la COVID-19 en la gestación}

En una cohorte de 91 mujeres diagnosticadas de COVID-19 en el periodo gestacional o en el posparto se determinó que $42(46.2 \%)$ requirieron hospitalización por COVID-19, mientras que entre las que dieron a luz al momento del estudio (23 pacientes con infección activa al llegar al parto) la prematuridad fue del $34.8 \%{ }^{16}$. Un estudio realizado en Suecia determinó que las mujeres gestantes positivas para SARS-CoV-2 presentaron mayor prevalencia de preeclampsia $(7.7 \%)$ que las mujeres gestantes negativas para el test viral (4.3\%), sin encontrar diferencias significativas en cuanto a la prematuridad ( $9 \%$ vs. $7.5 \%)^{17}$. En contraste, el estudio multinacional de Villar, et al. ${ }^{14}$ reporta un aumento de partos pretérmino en pacientes con diagnóstico de COVID-19 (22\%) en comparación con un grupo control (13.6\%). Las diferencias pueden deberse al tamaño de la población analizada y a las características de esta, considerando el riesgo asociado a la población de origen latino o hispano ${ }^{12}$.

En cuanto a la necesidad de hospitalización, en un reporte de 8207 mujeres gestantes y 83,205 mujeres no gestantes infectadas con SARS-CoV-2 se determinó que la tasa de hospitalización fue del $31.5 \%$ en las gestantes y del $5.8 \%$ en las no gestantes ${ }^{12}$. Estas cifras de hospitalizaciones muestran que las mujeres gestantes tienen una mayor prevalencia de cuadros graves o críticos de COVID-19, especialmente si presentan comorbilidad como hipertensión, diabetes (pregestacional y gestacional) y obesidad ${ }^{14,18}$. Los reportes iniciales que indicaron un mayor riesgo de resultados obstétricos y perinatales adversos, junto con necesidad de tratamiento en la $\mathrm{UCl}$ en gestantes con diagnóstico de COVID-19 12,18,19, han sido confirmados por el estudio de Villar, et al. ${ }^{14}$ antes citado, en el cual se determinó 
que las mujeres gestantes con COVID-19 tuvieron un mayor riesgo de desarrollo de preeclampsia, eclampsia o síndrome HELLP (hemolysis, elevated liver enzymes and low platelet count), ingreso a $\mathrm{UCl}$, parto pretérmino y recién nacidos de bajo peso. Además, entre las 706 pacientes con COVID-19 estudiadas, el riesgo de mortalidad fue de un $1.6 \%$ (22 veces mayor que en las embarazadas sin COVID-19), lo que se corrobora por el análisis de información clínica de 400,000 pacientes embarazadas con sintomatología de COVID-19 atendidas durante el año 2020 en los Estados Unidos de América $^{13}$. Ambos reportes indican que las mayores gravedad y mortalidad se relacionan con desigualdades socioeconómicas que afectan las condiciones de vida y la atención de salud de la población vulnerable.

Por otro lado, más allá del desarrollo de cuadros graves o críticos de COVID-19, no existe certeza sobre los potenciales efectos del virus sobre el proceso gestacional, inclusive en pacientes asintomáticas. Una forma de evaluar estos posibles efectos es mediante el estudio de la placenta. Además, la posible transmisión vertical del virus desde la madre hacia el feto, in utero, ha sido un tema de preocupación desde el comienzo de la pandemia. Si bien no existe una conclusión al respecto, la evidencia publicada muestra que la transmisión vertical es plausible, aunque no sería una vía frecuente de infección ${ }^{20-22}$. En los siguientes párrafos recopilamos la evidencia sobre la presencia de SARS-CoV-2 en la placenta y sus potenciales efectos en la función placentaria, así como los mecanismos de acción propuestos.

\section{Estudios sobre SARS-CoV-2 en la placenta}

Entre los artículos publicados sobre COVID-19 y placenta destacan los estudios que reportan directamente la detección de SARS-CoV-2 en la placenta (Tabla 1). Estos reportes utilizan, principalmente, pruebas de reacción en cadena de la polimerasa en tiempo real (qPCR), inmunohistoquímica (IHQ), hibridación in situ (HIS) y microscopía electrónica para la detección del virus o parte de él. En el estudio de Smithgall, et al. ${ }^{23}$ se realizó el análisis de placenta de 51 pacientes con test positivo para SARS-CoV-2. En este trabajo no se detectó el SARS-CoV-2 por IHQ ni por HIS en la placenta de mujeres SARS-CoV-2 positivo, ya fueran sintomáticas 0 asintomáticas, comparadas con el grupo control. De manera similar, el estudio de Hecht, et al. ${ }^{24}$ reporta una baja positividad (2 de 19 casos) de infección por SARS-CoV-2 en placentas de madres diagnosticadas con COVID-19, mientras que en el estudio de Hosier, et al. ${ }^{25}$ se determinó la presencia tanto de RNA como de proteínas del SARS-CoV-2 en la placenta. Además, diversos estudios de casos informan de la presencia de ARN del SARS-CoV-29,24,26-35, de proteína nucleocápside SARS ${ }^{24,34-38} 0$ de partículas virales $^{37,39}$. De manera relevante, se ha reportado la presencia de SARS-CoV-2 principalmente en el sincitiotrofoblasto $24,25,27,31,34,35,38,40$, mientras que un par de estudios han evidenciado la presencia del virus en el endotelio fetal ${ }^{36,41}$. Llama la atención que la detección del virus en el endotelio fetoplacentario ha sido en pacientes asintomáticas para COVID-19, una característica que deberá ser corroborada con más estudios.

Las evidencias resumidas en la tabla 1 muestran que la infección placentaria por SARS-CoV-2 es factible, por lo que la posibilidad de que exista transmisión vertical desde la madre hacia el feto es un aspecto sobre el cual hay que poner atención. En el estudio de Fenizia, et al. ${ }^{21}$ se detectó la presencia del virus tanto en la placenta como en una muestra nasofaríngea del recién nacido en dos casos. En ambos se hallaron anticuerpos anti-SARS-CoV-2 en el plasma materno y en un caso se detectaron anticuerpos en la sangre de cordón umbilical. En el caso reportado por Morotti, et al. ${ }^{38}$ se detectó la presencia del SARS-CoV-2 en la placenta de una gestación en la que tanto la madre como el recién nacido resultaron positivos en el test nasofaríngeo. De acuerdo con esta evidencia, la transmisión vertical del SARS-CoV-2 parece ser factible, aunque sería un evento de baja frecuencia. Esta conclusión se apoya en el estudio multicéntrico de Villar, et al. $^{14}$, que determinó que en 416 neonatos de madres diagnosticadas con COVID-19 que fueron sometidos a test para SARS-CoV-2 resultaron positivos un $12.9 \%$.

En cuanto a la relación entre infección placentaria y compromiso fetal, la detección de SARS-CoV-2 en dos placentas de casos de muerte fetal alerta sobre la posibilidad de que la infección placentaria pueda relacionarse con deterioro funcional placentario por una reacción hiperinflamatoria ${ }^{33}$. Otro par de estudios de caso documentan aborto espontáneo (19 semanas de gestación $)^{26} 0$ aborto inducido (22 semanas de gestación $)^{25}$ con cuadros respiratorios maternos graves e infección viral placentaria, sin detección del virus en el líquido amniótico ni en los tejidos fetales ${ }^{25,26}$. Todos estos casos ocurrieron durante el segundo trimestre de gestación en mujeres con sobrepeso, obesidad o preeclampsia. Por otro lado, un estudio con 21 placentas de mujeres con test positivo para SARS-CoV-2 que 
Tabla 1. Resumen de artículos que estudian la presencia de SARS-CoV-2 en la placenta

\begin{tabular}{|c|c|c|c|c|c|c|c|c|}
\hline \multicolumn{4}{|c|}{ Método detección en placenta } & \multicolumn{2}{|c|}{ Síntomas de COVID-19 } & n & País & Ref \\
\hline $\begin{array}{r}\text { qPCR } \\
\text { Neg } \\
\text { Neg }\end{array}$ & $\begin{array}{l}\text { IHO } \\
\text { ND } \\
\text { ND }\end{array}$ & $\begin{array}{l}\text { HIS } \\
\text { ND } \\
\text { ND }\end{array}$ & $\begin{array}{l}\text { ME } \\
\text { ND } \\
\text { ND }\end{array}$ & $\begin{array}{l}\text { Sí } \\
X \\
X\end{array}$ & No & $\begin{array}{l}1 \\
1\end{array}$ & $\begin{array}{l}\text { Turquía } \\
\text { China }\end{array}$ & $\begin{array}{l}63 \\
64\end{array}$ \\
\hline $\begin{array}{l}\text { Pos (3) Neg (8) } \\
\text { ND } \\
\text { ND } \\
\text { ND } \\
\text { Pos }\end{array}$ & $\begin{array}{l}\text { ND } \\
\text { ND } \\
\text { Neg (18) Pos (1) } \\
\text { Pos } \\
\text { ND }\end{array}$ & $\begin{array}{l}\text { ND } \\
\text { ND } \\
\text { Neg (17) Pos (2) } \\
\text { ND } \\
\text { ND }\end{array}$ & $\begin{array}{l}\text { ND } \\
\text { Pos } \\
\text { ND } \\
\text { ND } \\
\text { ND }\end{array}$ & $\begin{array}{c}X \\
X \\
N D \\
X\end{array}$ & $\begin{array}{c}\text { ND } \\
X\end{array}$ & $\begin{array}{c}11 \\
1 \\
19 \\
1 \\
1\end{array}$ & $\begin{array}{l}\text { EE.UU. } \\
\text { EE.UU. } \\
\text { EE.UU. } \\
\text { EE.UU. } \\
\text { India }\end{array}$ & $\begin{array}{l}32 \\
39 \\
24 \\
36 \\
30\end{array}$ \\
\hline $\begin{array}{l}\text { Pos (2) Neg (1) } \\
\text { ND } \\
\text { Pos } \\
\text { ND } \\
\text { ND } \\
\text { Pos } \\
\text { Pos } \\
\text { ND } \\
\text { Pos } \\
\text { Pos } \\
\text { Pos }\end{array}$ & $\begin{array}{l}\text { ND } \\
\text { Neg } \\
\text { Pos } \\
\text { Pos } \\
\text { Pos } \\
\text { ND } \\
\text { Pos } \\
\text { Pos } \\
\text { ND } \\
\text { ND } \\
\text { ND }\end{array}$ & $\begin{array}{l}\text { ND } \\
\text { Neg } \\
\text { ND } \\
\text { ND } \\
\text { Pos } \\
\text { ND } \\
\text { Pos } \\
\text { Pos (2) Neg (1) } \\
\text { ND } \\
\text { ND } \\
\text { ND }\end{array}$ & $\begin{array}{l}\text { ND } \\
\text { ND } \\
\text { ND } \\
\text { Pos } \\
\text { ND } \\
\text { ND } \\
\text { Pos } \\
\text { ND } \\
\text { ND } \\
\text { ND } \\
\text { ND }\end{array}$ & $\begin{array}{l}2 \\
50 \\
N D \\
X \\
X \\
X \\
X \\
X \\
X \\
X \\
X\end{array}$ & $\begin{array}{c}1 \\
26 \\
N D \\
X \\
X\end{array}$ & $\begin{array}{c}3 \\
76 \\
1 \\
1 \\
2 \\
1 \\
1 \\
3 \\
3 \\
1 \\
1\end{array}$ & $\begin{array}{l}\text { Brasil } \\
\text { EE.UU. } \\
\text { EE.UU. } \\
\text { EE.UU. } \\
\text { EE.UU. } \\
\text { Italia } \\
\text { EE.UU. } \\
\text { Italia } \\
\text { China } \\
\text { Suiza } \\
\text { Canadá }\end{array}$ & $\begin{array}{l}33 \\
23 \\
34 \\
37 \\
27 \\
28 \\
25 \\
31 \\
65 \\
26 \\
29\end{array}$ \\
\hline $\begin{array}{l}\text { Pos (2) Neg (29) } \\
\text { ND } \\
\text { ND } \\
\text { ND } \\
\text { Neg } \\
\text { Neg }\end{array}$ & $\begin{array}{l}\text { ND } \\
\text { ND } \\
\text { Pos } \\
\text { Pos } \\
\text { ND } \\
\text { ND }\end{array}$ & $\begin{array}{l}\text { ND } \\
\text { Neg } \\
\text { Pos } \\
\text { Pos } \\
\text { ND } \\
\text { ND }\end{array}$ & $\begin{array}{l}\text { ND } \\
\text { ND } \\
\text { ND } \\
\text { ND } \\
\text { ND } \\
\text { ND }\end{array}$ & $\begin{array}{c}X \\
15 \\
X \\
2 \\
11 \\
5\end{array}$ & $\begin{array}{c}32 \\
\\
3 \\
0 \\
19\end{array}$ & $\begin{array}{c}31 \\
47 \\
1 \\
5 \\
11 \\
24\end{array}$ & $\begin{array}{l}\text { Italia } \\
\text { EE.UU. } \\
\text { Italia } \\
\text { EE.UU. } \\
\text { México } \\
\text { Turquía }\end{array}$ & $\begin{array}{l}21 \\
48 \\
38 \\
35 \\
50 \\
42\end{array}$ \\
\hline
\end{tabular}

HIS: hibridación in situ; IHQ: inmunohistoquímica; ND: no determinado; Neg: negativo; Pos: positivo; qPCR: reacción en cadena de la polimerasa en tiempo real.

sufrieron pérdida temprana de la gestación $(<24$ semanas) no encontró ARN viral en la placenta ${ }^{42}$. A esta evidencia se suma un estudio con 100 casos de aborto espontáneo (y 125 controles, gestaciones que siguieron su curso) durante el primer trimestre de gestación, en el que no se hallaron diferencias significativas entre casos y controles para la detección de SARS-CoV-2 en una muestra nasofaríngea ${ }^{43}$. En estos últimos estudios se consigna que la mayoría de los casos fueron asintomáticos o con manifestaciones moderadas de COVID-19. Entonces, el compromiso fetal que pueda conducir a un aborto parece estar relacionado con la gravedad del cuadro materno. Así se informa en un estudio multinacional con 887 gestantes con resultado positivo para test de SARSCoV-2, en el cual se determinó un aumento de partos prematuros y de abortos espontáneos (antes de las 22 semanas) en el grupo de mujeres con embarazos de alto riesgo ${ }^{44}$. A su vez, el riesgo relativo de cuadros neonatales y perinatales graves, y la estancia en la $\mathrm{UCl}$ neonatal por más de 7 días, son considerablemente mayores en los recién nacidos con test positivo para SARS-CoV-2 ${ }^{14}$.

\section{Alteraciones vasculares placentarias asociadas con el SARS-CoV-2}

En cuanto a las alteraciones estructurales de la placenta, los hallazgos más significativos apuntan a que la infección por SARS-CoV-2 induce una mala perfusión útero-placentaria, con presencia de infartos placentarios, formación de ateromas en los vasos de la decidua, corioangioma y edema en las vellosidades placentarias $^{45}$. En el estudio de Mulvey, et al. ${ }^{46}$ se detectaron trombosis de vasos de la placa coriónica y disminución significativa de los capilares en las vellosidades coriónicas, lo que confirma la mala perfusión placentaria que podría asociarse con la infección viral. En el estudio de Baergen y Heller ${ }^{47}$, con 20 pacientes SARS-CoV-2 positivas (sintomáticas y asintomáticas), también se asocia la infección viral con una mayor propensión a la trombosis de vasos fetales y una menor vascularización en las vellosidades coriónicas. Un estudio con placentas de 44 casos positivos de SARS-CoV-2 y 44 casos negativos reportó mala perfusión vascular materna en el $36 \%$ de los casos positivos, frente al $18 \%$ de los casos negativos ${ }^{48}$. Por el contrario, un estudio de análisis histológico de placentas de 21 pacientes 
SARS-CoV-2 positivas (sintomáticas y asintomáticas) no detectó diferencias estructurales significativas en comparación con 20 placentas de pacientes con test de SARS-CoV-2 negativo ${ }^{49}$. Hay que considerar que en este último estudio no se determinó si las placentas estudiadas estaban infectadas con el virus ni si expresaban marcadores inflamatorios. Este último aspecto es relevante de acuerdo con el estudio de Fenizia, et al. ${ }^{21}$, en el cual se demuestran aumentos significativos de los marcadores de la inflamación, como la interleucina $1 \beta$ y la interleucina 6 , entre otras, tanto en la placenta como en el plasma materno de dos pacientes cuyas placentas presentaban infección por SARS-CoV-2. Se ha reportado que la presencia de SARS-CoV-2 en la placenta podría relacionarse con una respuesta inflamatoria en el espacio intervelloso (intervellositis histiocítica) y en las vellosidades coriónicas (vellositis), con presencia de macrófagos (CD68 y CD163) y de linfocitos T (CD3) en el espacio intervelloso ${ }^{23-25,29,31,34,36-38}$. La evidencia del aumento de citocinas proinflamatorias placentarias en presencia del virus, junto con la detección de macrófagos y alteraciones de tipo inflamatorio en los vasos placentarios, permiten proponer que el SARS-CoV-2 podría inducir vasculopatía placentaria y alteraciones de la perfusión materno-fetal.

Entre los estudios celulares y moleculares cabe destacar el trabajo de Flores-Pliego, et al..$^{50}$, en el que se describe que en las placentas de mujeres con COVID19 hay un aumento de la expresión del factor von Willebrand ( $f v W$ ) en las células endoteliales, junto con una disminución en la expresión endotelial de proteínas de adhesión celular VE-cadherina y claudina-5 en los casos de COVID-19 grave. El aumento del fvW se asociaría con un mayor riesgo de trombosis en los vasos placentarios, mientras que la disminución de las proteínas de adhesión se relacionaría con disfunción endotelial asociada al proceso inflamatorio placentario detectado en los casos graves de COVID-1950. El fvW es una glucoproteína plasmática sintetizada por las plaquetas y las células endoteliales que tiene un papel relevante en la hemostasia primaria, y su aumento en plasma sirve de indicador inflamatorio. Las concentraciones plasmáticas elevadas de fvW se han asociado con enfermedad coronaria e infarto ${ }^{51}$, siendo liberado en exceso cuando se produce daño endotelial por estados hiperinflamatorios ${ }^{52}$. Se ha reportado que las concentraciones plasmáticas de fvW estaban significativamente elevadas en los pacientes de COVID-19 que requirieron atención en la $\mathrm{UCl}$, en comparación con los pacientes con cuadros leves de la enfermedad ${ }^{53}$. Estos hallazgos se asocian con un aumento de la relación
sFIt-1/PIGF (sFlt-1: soluble fms-like tyrosine kinase 1; PIGF: placental growth factor) detectada en pacientes con neumonía por COVID-19, en comparación con pacientes con neumonía por otras causas ${ }^{54}$. El aumento de la tasa sFIt-1/PIGF ha sido determinado como un biomarcador de disfunción endotelial inducida por la preeclampsia, por lo que se ha propuesto que los cuadros de COVID-19 grave o crítica generarían un síndrome tipo preeclampsia ${ }^{55}$ que induciría daño endotelial. Por lo tanto, es factible pensar que en los cuadros graves de COVID-19 se produzca un daño endotelial sistémic ${ }^{56}$, incluyendo los vasos sanguíneos placentarios, debido al estado hiperinflamatorio. En la placenta, el daño endotelial sería parte del mecanismo que lleva al desarrollo de trombosis, infartos y remodelación de la pared vascular en los vasos sanguíneos de las vellosidades coriónicas y la decidua.

\section{Potencial mecanismo de infección placentaria por SARS-CoV-2}

Se ha determinado que el mecanismo de entrada a las células del SARS-CoV-2 requiere la expresión en la membrana plasmática de la enzima convertidora de la angiotensina 2 (ACE2), que actúa como receptor al unirse a la proteína viral spike ${ }^{57}$, mientras que la propagación del virus en el organismo depende también de la proteasa de serina TMPRSS2 ${ }^{58}$.

Para determinar si el SARS-CoV-2 tiene la capacidad de infectar y atravesar la placenta es relevante determinar si ACE2 y TMPRSS2 se expresan en el tejido placentario, específicamente en el trofoblasto y el endotelio fetal. Al momento, la evidencia es controversial. Por un lado, un estudio con 44 placentas de mujeres positivas para SARS-CoV-2 determinó la expresión de ACE2 restringida al sincitiotrofoblasto, mientras que la expresión de TMPRSS2 se detectó en las células endoteliales de las vellosidades coriónicas ${ }^{59}$. En este estudio se destaca que no se detectó la presencia del virus en ninguna placenta estudiada (Tabla 1), por lo que se especula que la falta de colocalización de ACE2 y TMPRSS2 en el sincitiotrofoblasto 0 el endotelio vellositario se relacionaría con la ausencia de infección placentaria, y tampoco se detectó la presencia de células Hofbauer ${ }^{59}$. Por otro lado, un estudio transcriptómico (RNA-seq) en células aisladas de placenta muestra que existe coexpresión de ACE2 y TMPRSS2 en el sincitiotrofoblasto, el citotrofoblasto y el trofoblasto extravellositario, aunque muy baja si se compara con los niveles de expresión de receptores de citomegalovirus y virus Zika, patógenos que se ha 


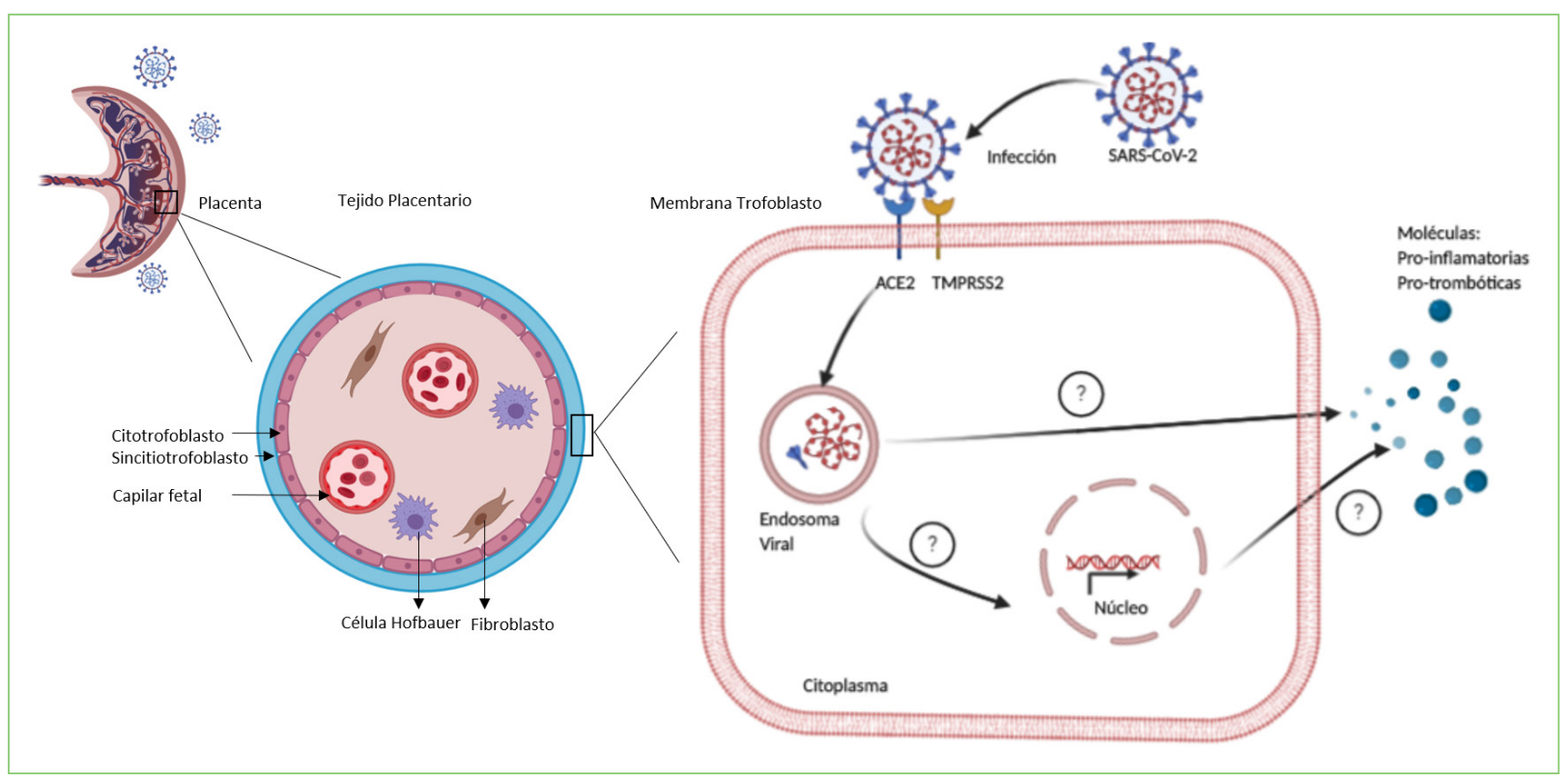

Figura 1. Potencial mecanismo de infección placentaria por SARS-CoV-2. La expresión de las proteínas ACE2 y TMPRSS2 en la membrana plasmática del sincitiotrofoblasto permite la unión del SARS-CoV-2 y su posterior endocitosis. Mediante mecanismos aún no descritos, la infección viral podría inducir un aumento en la síntesis y la secreción de moléculas proinflamatorias y protrombóticas, ya sea directamente desde el trofoblasto 0 como respuesta endotelial secundaria. La generación de estos procesos inflamatorios y trombóticos se correlaciona con la presencia de células de Hofbauer en el estroma vellositario, con alteraciones de la pared vascular de los capilares fetales y con el aumento de la expresión del factor von Willebrand en las células endoteliales.

comprobado que atraviesan la placenta y producen transmisión vertical hacia el feto ${ }^{59}$. Otro estudio transcriptómico en células aisladas de placenta muestra una alta expresión de ACE2 y TMPRSS2 en el sincitiotrofoblasto y el citotrofoblasto, aunque no se detecta expresión en el endotelio placentario ${ }^{60}$. Llama la atención que ambos estudios de transcriptoma en células únicas utilizan la misma base de datos (muestras de ArrayExpress E-MTAB-6701), pero el estudio de Li, et al. ${ }^{60}$ incluye una fuente de datos adicional de transcriptoma en células de trofoblasto. En la misma línea, se ha corroborado mediante RT-qPCR, HIS e inmunofluorescencia que ACE2 y TMPRSS2 se coexpresan en el trofoblasto placentario, mientras que están ausentes en el endotelio placentario ${ }^{61}$. Adicionalmente, un estudio anterior mostró que ACE2 se expresa en el sincitiotrofoblasto y el endotelio en la placenta de término, aumentando su expresión en el endotelio de la arteria umbilical en las pacientes con preeclampsia ${ }^{62}$. Igualmente se ha corroborado la expresión de ACE2 en el trofoblasto de placentas en las que también se ha detectado colonización por SARS-CoV-2 ${ }^{24,35}$. Por último, los ensayos de infección in vitro con pseudovirus SARS-CoV-2 en células de trofoblasto aisladas de placenta y línea celular placentaria JEG3 demuestran la capacidad infectiva del virus dada la afinidad de ACE2 y la proteína spike ${ }^{35,61}$. En la figura 1 proponemos que la colonización de la placenta por SARSCoV-2 es posible, en tanto restringida al sincitiotrofoblasto de las vellosidades coriónicas. La infección placentaria desencadenaría un mecanismo proinflamatorio y protrombótico que podría afectar a las células adyacentes, entre ellas las células endoteliales de los vasos sanguíneos fetales. La falta de coexpresión de ACE2 y TMPRSS2 en el endotelio fetal serviría de barrera para evitar el paso del SARS-CoV-2 a la sangre fetal, aunque en condiciones de daño endotelial (alteración de uniones intercelulares) podría darse esa situación.

\section{Conclusiones y direcciones futuras}

Luego de 1 año de avance de la pandemia de COVID-19 se ha podido establecer que las mujeres en gestación tienen mayor probabilidad de desarrollar cuadros graves o críticos de COVID-19, sobre todo si presentan comorbilidad como obesidad, hipertensión o diabetes. La evidencia muestra que la infección placentaria por SARS-CoV-2 es factible, posiblemente en relación con procesos proinflamatorios y daño en los vasos sanguíneos. Aunque la transmisión vertical sea 
poco frecuente, puede ocurrir y los recién nacidos positivos para SARS-CoV-2 presentan mayor riesgo de sufrir cuadros neonatales o perinatales graves.

En conclusión, la información disponible indica la mayor vulnerabilidad de las mujeres gestantes y de los fetos en el contexto de la pandemia de COVID-19. Es importante que se realice una mayor cantidad de estudios para comprender el real impacto de la pandemia en las mujeres gestantes y en los recién nacidos, incluyendo estudios de seguimiento de pacientes que tuvieron COVID-19 durante la gestación, para determinar efectos a medio y largo plazo. También queremos hacer hincapié en la necesidad de realizar estudios para describir los efectos del SARS-CoV-2 sobre mecanismos celulares y moleculares relevantes para la función de la placenta humana, para comprender los alcances tanto de este virus como de otros a los que se pueda ver expuesto el ser humano en el futuro. Estos estudios deberían incluir mecanismos relacionados con la función endotelial placentaria, como son los relacionados con la síntesis de moléculas vasoactivas, la regulación del estrés oxidativo y la integridad de la barrera endotelial, entre otros.

\section{Agradecimientos}

M. González agradece a Susana Araya y Salvador González por el apoyo cotidiano, especialmente durante la pandemia.

\section{Financiamiento}

C. Escudero es apoyado por Fondecyt 1200250. M. González es apoyado por proyecto VRID 2020000157MUL. C. Escudero es apoyado por proyecto FONDECYT 1200250.

\section{Conflicto de intereses}

Los autores declaran que no tienen conflictos de intereses.

\section{Responsabilidades éticas}

Protección de personas y animales. Los autores declaran que para esta investigación no se han realizado experimentos en seres humanos ni en animales.

Confidencialidad de los datos. Los autores declaran que en este artículo no aparecen datos de pacientes.
Derecho a la privacidad y consentimiento informado. Los autores declaran que en este artículo no aparecen datos de pacientes.

\section{Bibliografía}

1. Ciotti M, et al. COVID-19 outbreak: an overview. Chemotherapy. 2019;64:215-23.

2. Mitchell C. OPS/OMS I La OMS caracteriza a COVID-19 como una pandemia. Pan American Health Organization/World Health Organization. Disponible en: https://www.paho.org/hq/index.php?option=com_content\&view=article\&id=15756:who-characterizes-covid-19-as-a-pandemic\&ltemid=1926\&lang=es (2020).

3. Timeline: WHO's COVID-19 response. Disponible en: https://www.who. int/emergencies/diseases/novel-coronavirus-2019/interactive-timeline.

4. DEIS. Gob.cl - Cifras oficiales. Gobierno de Chile. Disponible en: https:// www.gob.cl/coronavirus/cifrasoficiales/.

5. Defunciones Semanales 1.1 - DEIS I Tableau Public. Disponible en: https://public.tableau.com/profile/deis4231\#!/vizhome/DefuncionesSemanales1_1 16102396688650/DEF?publish=yes.

6. Embarazadas y COVID-19 en Chile - SOCHOG. Disponible en: https:// sochog.cl/archivos/14897.

7. Hegewald MJ, Crapo RO. Respiratory physiology in pregnancy. Clin Chest Med. 2011;32:1-13.

8. Fu Q. Hemodynamic and electrocardiographic aspects of uncomplicated singleton pregnancy. Adv Exp Med Biol. 2018;1065:413-31.

9. Cheng SO, Khan S, Alsafi Z. Maternal death in pregnancy due to COVID-19. Ultrasound Obstet Gynecol. 2020;56:122.

10. Takemoto MLS, et al. The tragedy of COVID-19 in Brazil: 124 maternal deaths and counting. Int J Gynaecol Obstet. 2020;151:154-6.

11. Lumbreras-Márquez MI, Campos-Zamora M, Leon HLD, Farber MK Maternal mortality from COVID-19 in Mexico. Int $\mathrm{J}$ Gynecol Obstet. 2020;150:266-7

12. Ellington $\mathrm{S}$, et al. Characteristics of women of reproductive age with laboratory-confirmed SARS-CoV-2 infection by pregnancy status - United States, January 22-June 7, 2020. MMWR Morb Mortal Wkly Rep. 2020:69:769-75.

13. Zambrano LD, et al. Update: Characteristics of symptomatic women of reproductive age with laboratory-confirmed SARS-CoV-2 infection by pregnancy status - United States, January 22-October 3, 2020. MMWR Morb Mortal Wkly Rep. 2020;69:1641-7.

14. Villar J, et al. Maternal and neonatal morbidity and mortality among pregnant women with and without COVID-19 infection: the INTERCOVID Multinational Cohort Study. JAMA Pediatr. 2021175:817-26.

15. Kimberlin DW, Stagno S. Can SARS-CoV-2 infection be acquired in utero? More definitive evidence is needed. JAMA. 2020;323:1788-9.

16. Barbero $P$, et al. SARS-CoV-2 in pregnancy: characteristics and outcomes of hospitalized and non-hospitalized women due to COVID-19. J Matern Fetal Neonatal Med. 2020;1-7.

17. Ahlberg M, et al. Association of SARS-CoV-2 test status and pregnancy outcomes. JAMA. 2020;324:1782-5.

18. Hernández O. COVID 19 y embarazo en Chile: informe preliminar del estudio multicéntrico GESTACOVID - SOCHOG. Disponible en: https:// sochog.cl/archivos/revista-documento/covid-19-y-embarazo-en-chile-informe-pr eliminar-del-estudio-multicentrico-gestacovid.

19. Collin J, Byström E, Carnahan A, Ahrne M. Public Health Agency of Sweden's Brief Report: Pregnant and postpartum women with severe acute respiratory syndrome coronavirus 2 infection in intensive care in Sweden. Acta Obstet Gynecol Scand. 20202;99:819-22.

20. Auriti C, et al. Vertical transmission of SARS-CoV-2 (COVID-19): are hypotheses more than evidences? Am J Perinatol. 2020;37(S 02):S31-8.

21. Fenizia $C$, et al. Analysis of SARS-CoV-2 vertical transmission during pregnancy. Nat Commun. 2020;11:5128.

22. Kotlyar A, et al. Vertical transmission of coronavirus disease 2019: a systematic review and meta-analysis. Am J Obstet Gynecol. 2021:224:35-53 e3.

23. Smithgall MC, et al. Third trimester placentas of SARS-CoV-2-positive women: histomorphology, including viral immunohistochemistry and in situ hybridization. Histopathology. 2020;77:994-9.

24. Hecht JL, et al. SARS-CoV-2 can infect the placenta and is not associated with specific placental histopathology: a series of 19 placentas from COVID-19-positive mothers. Mod Pathol. 2020;33:2092-103.

25. Hosier $\mathrm{H}$, et al. SARS-CoV-2 infection of the placenta. J Clin Invest. 2020;130:4947-53.

26. Baud $D$, et al. Second-trimester miscarriage in a pregnant woman with SARS-CoV-2 infection. JAMA. 2020;323:2198-200.

27. Best Rocha A, et al. Detection of SARS-CoV-2 in formalin-fixed paraffin-embedded tissue sections using commercially available reagents. Lab Invest. 2020;100:1485-9. 
28. Ferraiolo A, et al. Report of positive placental swabs for SARS-CoV-2 in an asymptomatic pregnant woman with COVID-19. Medicina (Kaunas) 2020;56:306.

29. Kirtsman $\mathrm{M}$, et al. Probable congenital SARS-CoV-2 infection in a neonate born to a woman with active SARS-CoV-2 infection. CMAJ. 2020;192:E647-50.

30. Kulkarni R, et al. Early-onset symptomatic neonatal COVID-19 infection with high probability of vertical transmission. Infection. 2021;49:339-43.

31. Patanè $L$, et al. Vertical transmission of COVID-19: SARS-CoV-2 RNA on the fetal side of the placenta in pregnancies with COVID-19 positive mothers and neonates at birth. Am J Obstet Gynecol MFM 2020;2:100145

32. Penfield CA, et al. Detection of SARS-COV-2 in placental and fetal membrane samples. Am J Obstet Gynecol MFM. 2020;2:100133.

33. Richtmann R, et al. Fetal deaths in pregnancies with SARS-CoV-2 infection in Brazil: a case series. Case Rep Womens Health. 2020;27:e00243.

34. Vivanti AJ, et al. Transplacental transmission of SARS-CoV-2 infection. Nat Commun. 2020:11:3572.

35. Verma S, et al. SARS-CoV-2 colonization of maternal and fetal cells of the human placenta promotes alteration of local renin-angiotensin system. Med (N Y). 2021;2:575-90.e5.

36. Hsu AL, et al. Placental SARS-CoV-2 in a pregnant woman with mild COVID-19 disease. J Med Virol. 2021;93:1038-44.

37. Sisman J, et al. Intrauterine transmission of SARS-CoV-2 infection in a preterm infant. Pediatr Infect Dis J. 2020;39:e265-7.

38. Morotti D, et al. Molecular pathology analysis of SARS-CoV-2 in syncytiotrophoblast and Hofbauer cells in placenta from a pregnant woman and fetus with COVID-19. Pathogens. 2021:10:479.

39. Algarroba GN, et al. Visualization of SARS-CoV-2 virus invading the human placenta using electron microscopy. Am J Obstet Gynecol. 2020;223:275-8.

40. Sisman J, et al. Intrauterine transmission of SARS-COV-2 infection in a preterm infant. Pediatr Infect Dis J. 2020;39:e265-7.

41. Sánchez J, et al. Severe acute respiratory syndrome coronavirus 2 detected in placentas of 2 coronavirus disease 2019-positive asymptomatic pregnant women-case report. AJOG Glob. Rep. 2021;1:100001.

42. Halici-Ozturk $F$, et al. Investigating the risk of maternal-fetal transmission of SARS-CoV-2 in early pregnancy. Placenta. 2021;106:25-9.

43. Cosma S, et al. Coronavirus disease 2019 and first-trimester spontaneous abortion: a case-control study of 225 pregnant patients. Am J Obstet Gynecol. 2021;224:391.e1-7.

44. D'Antonio F, et al. Maternal and perinatal outcomes in high vs low risk-pregnancies affected by SARS-COV-2 infection (phase-2): the WAPM (World Association of Perinatal Medicine) working group on $\mathrm{CO}$ VID-19. Am J Obstet Gynecol MFM. 2021;3:100329.

45. Shanes ED, et al. Placental pathology in COVID-19. Am J Clin Pathol. 2020;154:23-32.

46. Mulvey JJ, Magro CM, Ma LX, Nuovo GJ, Baergen RN. Analysis of complement deposition and viral RNA in placentas of COVID-19 patients. Ann Diagn Pathol. 2020;46:151530.
47. Baergen RN, Heller DS. Placental pathology in Covid-19 positive mothers: preliminary findings. Pediatr Dev Pathol. 2020;23:177-80.

48. Edlow AG, et al. Assessment of maternal and neonatal SARS-CoV-2 viral load, transplacental antibody transfer, and placental pathology in pregnancies during the COVID-19 pandemic. JAMA Netw. Open. 2020;3:e2030455

49. He $M$, et al. Histopathology of third trimester placenta from SARS-CoV-2-positive women. Fetal Pediatr Pathol. 2020;1-10.

50. Flores-Pliego A, et al. Molecular insights into the thrombotic and microvascular injury in placental endothelium of women with mild or severe COVID-19. Cells. 2021;10:364.

51. Huang S, Ninivaggi M, Chayoua W, de Laat B. VWF, platelets and the antiphospholipid syndrome. Int J Mol Sci. 2021;22:4200.

52. Katneni UK, et al. Coagulopathy and thrombosis as a result of severe COVID-19 infection: a microvascular focus. Thromb Haemost. 2020;120:1668-79.

53. Marco A, Marco P. Von Willebrand factor and ADAMTS13 activity as clinical severity markers in patients with COVID-19. J Thromb Thrombolysis. 2021;1-7.

54. Giardini V, et al. Increased SFLT-1/PIGF ratio in COVID-19: a novel link to angiotensin II-mediated endothelial dysfunction. Am J Hematol. 2020;95:E188-91.

55. Mendoza M, et al. Pre-eclampsia-like syndrome induced by severe COVID-19: a prospective observational study. BJOG Int J Obstet Gynaecol. 2020;127:1374-80.

56. Bonaventura $A$, et al. Endothelial dysfunction and immunothrombosis as key pathogenic mechanisms in COVID-19. Nat Rev Immunol. 2021;21:319-29.

57. Shang J, et al. Structural basis of receptor recognition by SARS-CoV-2 Nature. 2020;581: 221-4

58. Hoffmann M, et al. SARS-CoV-2 cell entry depends on ACE2 and TMPRSS2 and is blocked by a clinically proven protease inhibitor. Cell. 2020;181:271-80.e8.

59. Pique-Regi R, et al. Does the human placenta express the canonical cell entry mediators for SARS-CoV-2? Elife. 2020;9:e58716.

60. Li M, Chen L, Zhang J, Xiong C, Li X. The SARS-CoV-2 receptor ACE2 expression of maternal-fetal interface and fetal organs by single-cell transcriptome study. PloS One. 2020;15:e0230295.

61. Ouyang $Y$, et al. Term human placental trophoblasts express SARS-CoV-2 entry factors ACE2, TMPRSS2, and furin. mSphere. 2021:6:e0250-21.

62. Valdés $\mathrm{G}$, et al. Distribution of angiotensin-(1-7) and ACE2 in human placentas of normal and pathological pregnancies. Placenta. 2006;27:200-7.

63. Kalafat $E$, et al. Lung ultrasound and computed tomographic findings in pregnant woman with COVID-19. Ultrasound Obstet Gynecol. 2020;55:835-7.

64. Peng Z, et al. Unlikely SARS-CoV-2 vertical transmission from mother to child: a case report. J Infect Public Health. 2020:13:818-20.

65. Chen S, et al. [Pregnancy with new coronavirus infection: clinical characteristics and placental pathological analysis of three cases]. Zhonghua Bing Li Xue Za Zhi. 2020;49:418-23. 